\title{
BMJ Open Protocol for REducing Anti-Psychotic use in residential care-Huntington Disease (REAP-HD): a pilot cluster randomised controlled trial of a multifaceted intervention for health professionals
}

\author{
Clement T Loy, ${ }^{1,2}$ Andrew Hayen, ${ }^{3}$ Colleen McKinnon ${ }^{1}$
}

To cite: Loy CT, Hayen A, McKinnon C. Protocol for REducing Anti-Psychotic use in residential care-Huntington Disease (REAP-HD): a pilot cluster randomised controlled trial of a multifaceted intervention for health professionals. BMJ Open 2014;4:e006151. doi:10.1136/bmjopen-2014006151

- Prepublication history for this paper is available online. To view these files please visit the journal online (http://dx.doi.org/10.1136/ bmjopen-2014-006151).

Received 23 July 2014 Revised 4 November 2014 Accepted 6 November 2014

CrossMark

\footnotetext{
${ }^{1}$ Huntington Disease Service, Westmead Hospital, Westmead, New South Wales, Australia

${ }^{2}$ The University of Sydney, Sydney, New South Wales, Australia

${ }^{3}$ The University of New South Wales, Kensington, New South Wales, Australia

Correspondence to Dr Clement T Loy; clement.loy@sydney.edu.au behavioural symptoms), and (2) proportion of people with HD who have had a reduction in antipsychotic dosage at 4 months for each strategy, compared to 4 months prior to enrolment (to capture the possibility that both arms reduced antipsychotic use). Analysis will be by Intention-To-Treat and take into account the clustering. Recruitment is ongoing, as of July 2014.

Ethics and dissemination: This protocol has been approved by the Western Sydney Local Health District Human Research Ethics Committee, trial registration ACTRN12614000083695. Study results will be disseminated through peer-reviewed publication in an anonymous manner.
}

Introduction: Antipsychotics are commonly used for management of behavioural symptoms in dementia, among people in residential care. This continues to occur despite their modest effectiveness, potential harms including increased risk of death and stroke, and absence of detrimental effect when people with dementia were randomised to antipsychotic withdrawal. This study aims to test the hypothesis that the multifaceted REducing Anti-Psychotic use in residential care-Huntington Disease (REAP-HD) programme is more effective than standard staff education (SSE) in reducing antipsychotic use for people with $H D$ in residential care facilities (RCF).

Methods and analysis: this is a cluster randomised controlled trial with blinded outcome assessment. The study population is healthcare professionals looking after people with HD in individual RCF, in the state of New South Wales. Each RCF will be centrally randomised to the REAP-HD programme or the comparator, SSE. Blinded outcome assessment will be performed by examining drug charts and using the Neuropsychiatric Inventory-Q (NPI-Q). Primary outcome is the proportion of people with HD who have had a reduction in antipsychotic use 4 months after the intervention. Secondary outcome measures are (1) change in severity of behavioural symptoms, as measured by the NPI-Q at 4 months (to ensure antipsychotic reduction has not lead to worsening

Trial registration number: ACTRN12614000083695, the Australian New Zealand Clinical Trials Registry.

\section{INTRODUCTION}

Antipsychotics are commonly used for management of behavioural symptoms in dementia, among people in residential care in Australia. This continues to occur despite guidelines from Australia, ${ }^{1}$ the $\mathrm{UK}^{2}$ and the $\mathrm{USA}^{3}$ all recommending antipsychotics as second line, time-limited therapy subject to regular review. The evidence supporting these recommendations include: (1) modest benefit of antipsychotics from randomised controlled trial (RCT) data, ${ }^{4}$ (2) potential harm including increased risk of death ${ }^{6}$ and stroke, ${ }^{7}$ and (3) the absence of detrimental effects when people with dementia were randomised to antipsychotic withdrawal. ${ }^{8}$

In 2005, the US Food and Drug Administration (FDA) issued a Boxed Warning about the increased risk of death associated with off-label atypical antipsychotic use in this context, and a similar warning for conventional antipsychotics followed. ${ }^{9}$ Accordingly, from 1999 to 2007, antipsychotic use in dementia dropped from $18 \%$ to $15 \%$ in the USA. $^{10}$ In contrast, antipsychotic use among Sydney nursing home residents actually increased from $23 \%$ in 1998 , to $28 \%$ in $2009 .^{11}$ This represents a significant evidence-practice gap in Australia.

The importance of this evidence-practice gap has also been recognised in the UK recently through an independent report. ${ }^{12}$ It estimated that up to two-thirds of antipsychotics for people with dementia could be avoided if appropriate support were available, and the 
excess antipsychotic use could lead to an additional 1800 deaths and 1620 strokes in the UK per year.

Barriers to reducing antipsychotic use in Australian residential care facilities (RCF) include (1) the complexity of guidelines, (2) the disjunction between prescribers (medical staff) and carers facing the behavioural problems (nursing staff), and (3) absence of a system to ensure medication review and therefore unnecessary continuation of antipsychotics.

REducing Anti-Psychotic use in residential careHuntington Disease (REAP-HD) aims to overcome some of these barriers in a subgroup of people with dementia - people with HD. HD is an autosomal dominant, progressive, neurodegenerative disorder, due to abnormal CAG expansion in the chromosome 4 Huntingtin gene. ${ }^{13}$ It is one of the most common neurogenetic disorders, ${ }^{14}$ and has devastating consequences for patients and their families. ${ }^{15}$ Typical age of onset is in the $30 \mathrm{~s}-40 \mathrm{~s}$, and symptoms include involuntary movements, cognitive/ behavioural symptoms and psychiatric disorders. ${ }^{13}$ Our understanding of HD has evolved from a familial movement disorder to a multisystem, chronic disease requiring complex care. Cognitive impairment is very common in $\mathrm{HD},{ }^{16}$ and dementia in the context of HD is one of the most common reason for RCF admission. People with HD make up a significant proportion of the very young living in New South Wales nursing homes-10.2\% of people aged 50 or under in NSW nursing homes have HD (baseline statistics from the Young Person in Nursing Home National Alliance ${ }^{17}$ ). Since people with HD tend to be younger and have behavioural symptoms, anecdotally the prevalence of antipsychotic use is highalthough the exact figure is unknown.

\section{AIMS}

The primary aim of this study is to test the hypothesis that the multifaceted REAP-HD programme is more effective than standard staff education (SSE) in reducing antipsychotic use for people with HD in RCF, 4 months (120 days) after the intervention.

The two secondary aims are

- To test the hypothesis that behavioural symptoms at 4 months are not worsened after REAP-HD or SSE;

- To test the hypothesis that REAP-HD and SSE reduce antipsychotic use, compared to what was prescribed 4 months prior to enrolment.

\section{METHODS}

These hypotheses will be tested in a cluster RCT with blinded outcome assessment. The study population is healthcare professionals looking after people with HD in individual RCF in the state of New South Wales, Australia. The tested intervention (REAP-HD programme) will be centrally randomised against the comparator SSE. Blinded outcome assessment will be performed by examining drug charts and using the Neuropsychiatric Inventory-Q (NPI-Q). ${ }^{18}$
Study setting, eligibility criteria and recruitment

People with HD will be invited to join the study through two routes: in response to a notice in the newsletter of the HD NSW (peak patient organisation in our state), or via direct telephone invitation to the person with HD or his/her family using contact numbers in the HD Service/Hunter HD Service client database. Invitations will be presented using a standardised telephone script and carried out by a nurse experienced in the care of people with HD, and already known to the person with HD or his/her family through clinical care.

If the initial response to recruitment is positive, then the patient information/consent form will be sent to the person with HD and his/her family for detailed consideration before a final decision is made re consent. The recruiting nurse will assess competency of people with HD to consent. If deemed competent, the person with HD will be the primary person to consent, but the person's family will also be asked to act as witness on the consent form. If the person with HD is deemed not capable of providing informed consent, the guardian/ senior person responsible would be asked to sign the form, with the person with HD as witness if possible.

Once we have confirmed informed consent from the person with HD and his/her family, we will contact his/ her RCF using a standard letter of introduction. We will identify the Registered Nurse (RN) who is primarily responsible for the person with $\mathrm{HD}$, and confirm that the person satisfies the inclusion/exclusion criteria (box 1). An overall schema for the study can be found in table 1 .

The consent process involves people with HD (for collection of personal information), as well as the health professional looking after people with HD (for randomised interventions). Once the person with HD's eligibility is confirmed, the recruitment nurse will seek

\section{Box 1 Inclusion/exclusion criteria for REAP-HD}

Inclusion criteria

1. Male or female 18 years or older.

2. Clinical Huntington disease and a confirmatory family history $\mathrm{OR} \geq 36 \mathrm{CAG}$ repeats on genetic testing.

3. Living in a Residential Care Facility in NSW, including group homes with $24 \mathrm{~h}$ supervision, hostels and nursing homes.

4. Currently receiving a stable dose of regular antipsychotic medications for management of behavioural symptoms, for at least 4 months prior to enrolment (see exclusion criteria).

5. Able to provide informed consent, or have a suitable senior person responsible who is able to provide informed consent.

Exclusion criteria

1. Change of antipsychotic dose within 4 months prior to enrolment.

2. Psychotic symptoms (new hallucinations or delusions) within 1 year of enrolment.

3. People taking antipsychotic medications solely for control of chorea.

4. Other unstable medical or psychiatric illness, making it unsafe to reduce antipsychotic dose. 
Table 1 Overall study schema for REAP-HD

\begin{tabular}{|c|c|c|c|c|c|}
\hline & \multicolumn{5}{|l|}{ Study period } \\
\hline & \multirow[b]{2}{*}{$\begin{array}{l}\text { Enrolment } \\
\text { Pre-randomisation }\end{array}$} & \multirow[b]{2}{*}{$\begin{array}{l}\text { Off-site } \\
\text { randomi-sation }\end{array}$} & \multicolumn{2}{|l|}{ Post-allocation } & \multirow[b]{2}{*}{$\begin{array}{l}\text { Close-out } \\
4 \text { months }\end{array}$} \\
\hline & & & $\begin{array}{l}\text { In person } \\
\text { visit at time } 0\end{array}$ & $\begin{array}{l}\text { Follow-up phone } \\
\text { call at } 1 \text { month }\end{array}$ & \\
\hline \multicolumn{6}{|l|}{ Enrolment } \\
\hline $\begin{array}{l}\text { Recruitment of people with } \\
\text { Huntington disease/family }\end{array}$ & $\mathrm{X}$ & & & & \\
\hline $\begin{array}{l}\text { Informed consent from people } \\
\text { with Huntington disease/family }\end{array}$ & $x$ & & & & \\
\hline $\begin{array}{l}\text { Informed consent from health } \\
\text { professional at residential care } \\
\text { facility }\end{array}$ & $\mathrm{x}$ & & & & \\
\hline Allocation & & $\mathrm{x}$ & & & \\
\hline \multicolumn{6}{|l|}{ Interventions } \\
\hline REAP-HD & & & 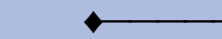 & $\longrightarrow$ & \\
\hline Standard staff education & & & $\diamond$ & $\longrightarrow$ & \\
\hline \multicolumn{6}{|l|}{ Assessments } \\
\hline $\begin{array}{l}\text { Collection of demographic data, } \\
\text { antipsychotic dosage } 4 \text { months } \\
\text { prior to enrolment }\end{array}$ & $x$ & & & & \\
\hline $\begin{array}{l}\text { Blinded collection of current } \\
\text { antipsychotic dosage }\end{array}$ & $x$ & $x$ & & & $x$ \\
\hline $\begin{array}{l}\text { Blinded telephone collection of } \\
\text { current NPI-Q score }\end{array}$ & & $\mathrm{x}$ & & & $x$ \\
\hline
\end{tabular}

consent from the $\mathrm{RN}$ or other appropriate representatives of the RCF, using the Health Professional Participant Information Consent Form. This form will also ask for permission from the health professional to be contacted for future studies. With informed consent, we will collect basic demographic data and medical history (including duration and mode of onset of HD) about the person with HD. With specific consent from the person with $\mathrm{HD}$, we will supplement this with data from their medical records. Team members will also collect some basic data about the residential facility (profit/non-profit, number of residents, number of residents with HD, whether there is a quality manager or pharmacist review, name and address of general practitioner (GP)).

They will also arrange a date for the implementation team members to visit the RCF (when the responsible $\mathrm{RN}$ will be present). The recruitment nurse will contact an off-site biostatistician (AH) to notify him of successful enrolment. The recruitment nurse will not be given the intervention allocation details at any stage of the study. This is to ensure blindedness since the recruitment nurse will also be the assessor for the primary and secondary outcomes.

\section{Interventions}

Since neither the implementation team nor the health professionals receiving the intervention can be blinded to the content of the intervention, we have incorporated a number of measures to maintain blinding. Outcome assessment will be blinded as above. Health professionals at the RCF will receive letters explaining the intent of REAP-HD in comparing implementation strategies, but the exact content of the interventions will be concealed. Thus health professionals will know that they have been randomised in one of two training programmes, but they will not know the content of the other programme, or whether the programme they have received is the 'new' intervention (REAP-HD) or SSE.

REAP-HD is a multifaceted intervention designed after barrier analysis with a number of nurses experienced in behavioural management in $\mathrm{HD}$, using the theoretical domain framework. ${ }^{19}$ A key feature of the programme is a clinical pathway for management of behavioural symptoms (figure 1). This clinical pathway targets skills and professional role by operationalising a strategy in delaying antipsychotic use whenever possible. It is hoped that by simplifying current behavioural management guidelines it will empower nursing staff to pursue nonpharmacological intervention and delay calling the GP for a drug order. It also incorporates a reminder to review and reduce antipsychotic dosage, as an aid to focus attention in the decision process (figure 2). In the REAP-HD programme, the intervention team will visit the RCF and deliver a 45-minute Powerpoint presentation. This focuses on antipsychotic use and the clinical pathway, rather than being just a general introduction to HD. The Director of Nursing, Deputy Director of Nursing, Nurse Educator, Nursing Unit Manager, as well as the ward nursing staff (RN, Endorsed Enrolled Nurses and Assistants in Nursing) will all be invited. The RCF will be given copies of the clinical pathway to incorporate 
Figure 1 REducing

Anti-Psychotic use in residential care-Huntington Disease clinical pathway. UTI, urinary tract infection.
Behavioural management clinical pathway for people with Huntington Disease Huntington Disease Service, Westmead, 2012

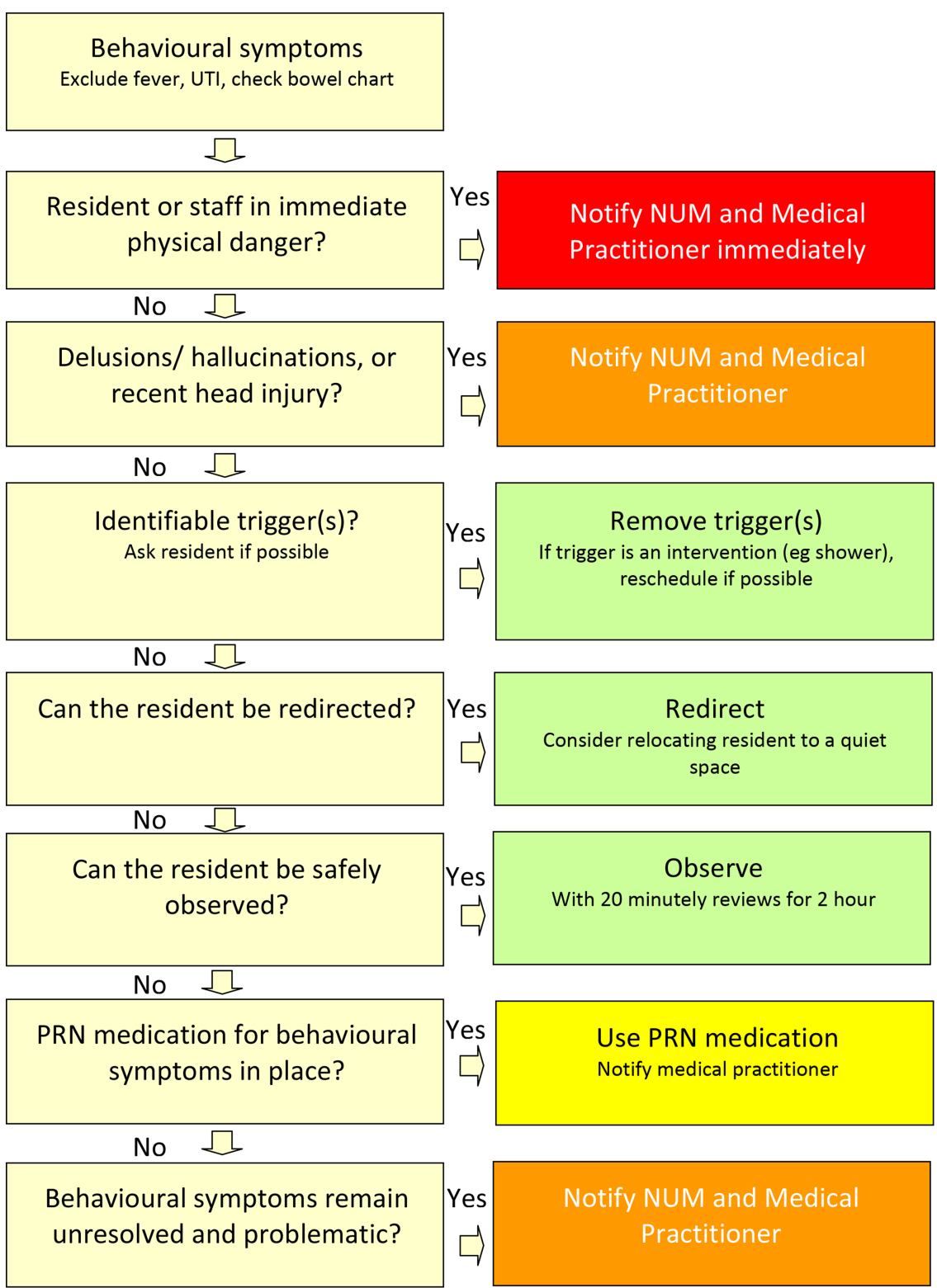

into the resident's notes/careplan. Banner pens (figure 3) with the REAP-HD logo and an abridged clinical pathway will be given to the RCF as reminders for the trial. Our intervention team will carry out a telephone reminder/follow-up call to the responsible RN 1 month after the intervention. At that juncture the $\mathrm{RN}$ will be asked about usage of the clinical pathway and any practical barriers to antipsychotic reduction. The GP will be sent a letter and the clinical pathway from the HD Service, explaining how the clinical pathway could be used for behavioural management. It can be argued that a face-to-face meeting with the GP would have been more effective. However, in terms of routine application of REAP-HD in the future outside of a trial setting, a letter is the only realistic option.

SSE comprises a different 45-minute Powerpoint presentation, presented in person. This presentation has been used by our Outreach Nurses for RCF nursing in-service over the past 5 years. This is designed to match REAP-HD in the number/duration of contact, but not content. The Director of Nursing, Deputy Director of Nursing, Nurse Educator, Nursing Unit Manager, as well as the ward nursing staff ( $\mathrm{RN}$, Endorsed Enrolled Nurses, and Assistants in Nursing) will all be invited. This represents current best practice and, in fact, many people with HD in RCF do not even benefit from an Outreach Education session like this. The SSE includes an introduction to HD and also some suggestions for behavioural management. The Powerpoint presentation will be updated with two additional slides on the modest efficacy and potential harm of antipsychotic use in this context. Our intervention team will carry out a telephone reminder/follow-up call to the responsible RN 1 month after the intervention. At 
Figure 2 REducing

Anti-Psychotic use in residential care-Huntington Disease medication review reminder.

Antipsychotic medications for behavioral symptoms in Dementia

- Modest benefit

- Potential harms including excess strokes and deaths

- Safe to withdraw

3-monthly reviews

\begin{tabular}{|c|c|c|c|c|}
\hline Month-Yr & $\begin{array}{l}\text { Regular } \\
\text { antipsychotic } \\
\text { dose }\end{array}$ & $\begin{array}{l}\text { PRN } \\
\text { antipsychotics } \\
\text { used }\end{array}$ & $\begin{array}{l}\text { Can } \\
\text { antipsychotic } \\
\text { dose be } \\
\text { reduced? }\end{array}$ & Comments \\
\hline & & & & \\
\hline & & & & \\
\hline & & & & \\
\hline & & & & \\
\hline & & & & \\
\hline & & & & \\
\hline & & & & \\
\hline & & & & \\
\hline & & & & \\
\hline & & & & \\
\hline & & & & \\
\hline & & & & \\
\hline
\end{tabular}

that juncture the $\mathrm{RN}$ will be asked about any practical barriers to antipsychotic reduction. The GP will also be sent a letter about the purpose of the trial but not details of the interventions.

\section{Outcome measurement}

The primary outcome measure for the RCT is the proportion of people with HD who have had a reduction in antipsychotic use, 4 months after the education session. Before the intervention, each RCF will be asked to fax us the medication chart (regular and prn) for the week preceding the planned intervention and the medication chart 4 months (120 days) prior to that. Similarly, 4 months after the intervention the RCF will also be asked to fax over a medication chart. These charts will be assessed and total antipsychotic dosage recorded by the research team member who is blinded to allocation of the intervention. If there has been a change in the type of antipsychotic used, then dose equivalence will be calculated using Woods. ${ }^{20}$

There are two secondary outcome measures. First is the change in severity of behavioural symptoms, as measured by the NPI-Q. ${ }^{18}$ This is an important safety measure to ensure that antipsychotic reduction does not lead to worsening behavioural symptoms. Our blinded assessor will interview the responsible $\mathrm{RN}$ via the phone on the day of the intervention and 4 months after, using the NPI-Q. The NPI-Q is a validated informant-based instrument measuring psychiatric and behavioural symptoms, and carer distress, in the preceding 4 weeks. In order to streamline the process, we will only be using the symptoms section of the questionnaire.

Second is the proportion of people with HD who have had a reduction in antipsychotic dosage at 4 months for each strategy, compared to 4 months prior to enrolment. It is possible that both interventions are effective-in 


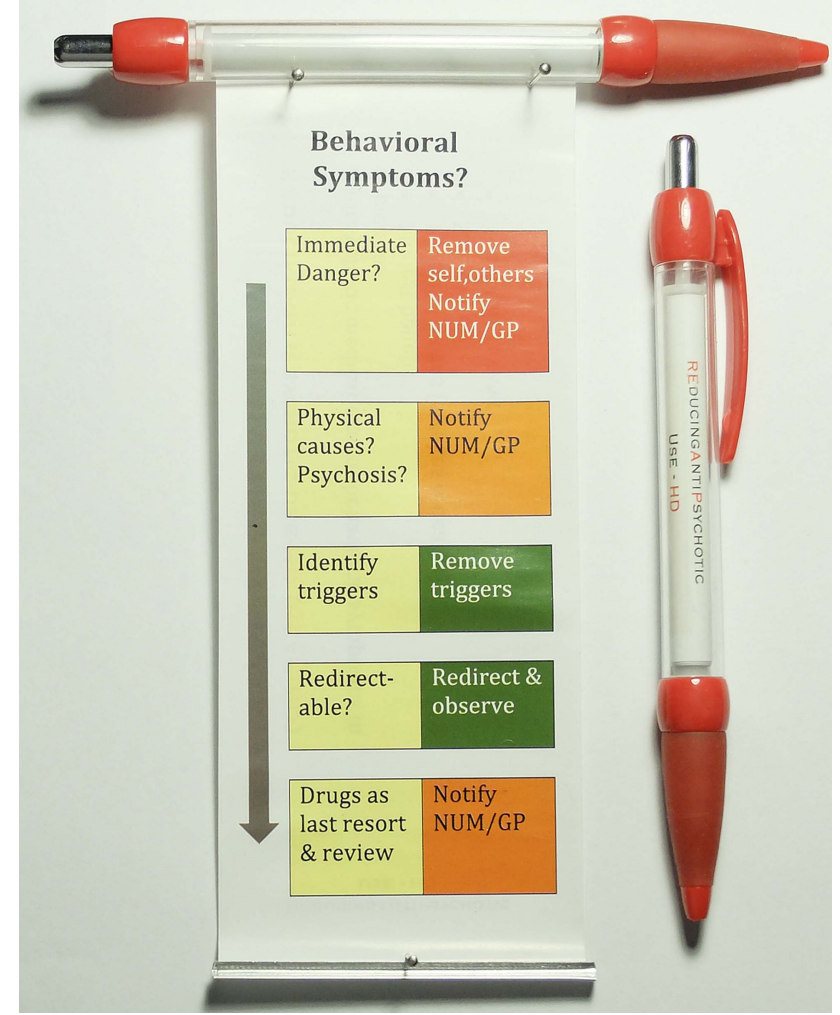

Figure 3 REducing Anti-Psychotic use in residential care-Huntington Disease Banner pens. GP, general practitioner.

which case the primary outcome may not be significantly different for the two arms, yet implementation of either strategy would still be effective in changing clinical practice. This secondary aim helps capture this possibility. This will only provide a before-after comparison, and it is always possible that some other external factor has led to reduction in antipsychotic use in both groups. However, stable antipsychotic dosage from 4 months pre-enrolment until enrolment, followed by a reduction in antipsychotic dosage postimplementation, would support the notion that the interventions had contributed towards antipsychotic reduction.

\section{Assignment of interventions and blinding}

An external off-site biostatistician (AH) will only notify the intervention team members of the allocation of intervention according to a 1:1 randomisation plan 1 working day before the appointed time of the RCF visit. As indicated above, the recruitment nurse will be blinded to group assignment and also act as the blinded outcome assessor. Study participants cannot be blinded to the intervention they receive but they are blinded to what the alternate intervention is, and whether they are receiving the 'new' intervention or the control SSE.

\section{Data management and analysis}

All data collected as part of this study will be stored securely under lock and key for paper records and under password protection for electronic records. Each participant will be allocated a reidentifiable participant number. Electronic data for Data Safety Monitoring Board (DSMB) and final statistical analysis will only be provided in a coded manner. Direct access to identifiable data will only occur for regulatory reasons, as detailed in the standard consent form for Western Sydney Local Health District.

Since there are only a small number of RCF with more than one HD resident, and cluster size is not uniform across RCF, it will be difficult to take into account clustering. For sample size calculation, we will therefore only 'count' one resident from each of RCF. Difference in primary outcome (proportion of people with HD who have had a reduction in antipsychotic use) between the two arms of the RCT will be expressed in terms of absolute risk reduction and relative risk reduction. Statistical significance between the two proportions will be tested with a $\chi^{2}$ test $(\mathrm{p}<0.05)$.

The likely effect size for REAP-HD or SSE on the primary outcome is unknown. This trial is therefore designed as a pilot study, with 19 participants in each arm. This represents approximately $30 \%$ of nursing homes in NSW looking after people with HD. Anecdotally, we have not seen any antipsychotic reduction in RCF following our previous education sessions. So assuming that antipsychotics will be reduced in $5 \%$ of people in the SSE arm, our sample size will be able to detect a difference of $50 \%$ versus $5 \%$ in the primary outcome for REAP-HD versus SSE, with a power of $82 \%$ $(\alpha=0.05)$. Whether this difference is achievable or not will be reassessed after this pilot trial. Changes in NPI-Q will be analysed using the paired t test. All analysis will be carried out on an Intention-To-Treat basis. Statistical comparison of the two intervention arms will be blinded from the identity of each arm. If there are additional residents available at a RCF (ie, more than one person with HD participating at a RCF), they can also be included in the study and the final statistical analysis will take into account the clustering by using logistic regression with general estimating equation.

\section{Monitoring}

The main adverse event is worsening of behavioural symptoms. A DSMB has been set up comprising our biostatistician and two neurologists with expertise in HD who are not involved in the trial. The DSMB is scheduled to review data once $50 \%$ of intended participants have completed the trial. The DSMB is set up to stop the trial under two conditions: (1) If the behavioural symptoms worsen in conjunction with a reduction of antipsychotic dose. Generally speaking a clinically meaningful change is defined as $30 \%$ change in the NPI-Q score. However, if smaller changes in critical domains have led to clinically adverse outcomes, the DSMB will be allowed to vary the threshold. (2) If there is adequate evidence that REAP-HD is superior to SSE in terms of the primary outcome. 


\section{ETHICS AND DISSEMINATION}

Trial protocol is V.4, 9 September 2013. Results of this trial will be communicated to the scientific community via conference presentations and journal publication(s). All data will be reported in an anonymous manner only. RCF, health professionals, people with $\mathrm{HD}$ and their families can all opt in for a summary of the trial results in clear understandable language. All RCF and health professionals, regardless of randomisation group, can also request a copy of the clinical pathway after completion of the trial. Results from this pilot study will provide an estimate of effect size for the REAP-HD intervention and intraclass correlation for cluster-randomised sites. These measures will help guide further implementation and planning of a larger cluster RCT.

Contributors CTL and CM were involved in the protocol, conceptualisation and development; $\mathrm{AH}$ was involved in the protocol development and statistical input. CTL and CM will be carrying out the study, together with another research nurse who will act as the blinded outcome assessor. AH will provide centralised off-site randomisation, and chair the Data and Safety Monitoring Board. All three authors drafted this protocol.

Funding The Australian National Health \& Medical Research Council Translating Research Into Practice Fellowship APP1035429.

Competing interests None.

Ethics approval This study has been peer-reviewed and approved scientifically by the Scientific Advisory Committee and ethically by the Human Research Ethics Committee of the Western Sydney Local Health District (HREC2012/6/4.8(3535) AU RED HREC/12/WMEAD/197). It is registered with the Australian New Zealand Clinical Trials Registry (ACTRN12614000083695).

Provenance and peer review Not commissioned; internally peer reviewed.

Data sharing statement No additional data are available.

Open Access This is an Open Access article distributed in accordance with the Creative Commons Attribution Non Commercial (CC BY-NC 4.0) license, which permits others to distribute, remix, adapt, build upon this work noncommercially, and license their derivative works on different terms, provided the original work is properly cited and the use is non-commercial. See: http:// creativecommons.org/licenses/by-nc/4.0/

\section{REFERENCES}

1. National Prescribing Service. Role of antipsychotics in managing behavioural and psychological symptoms of dementia. NPS Prescribing Practice Review, 2007.
2. National Institute for Health and Clinical Excellence. Dementia: supporting people with dementia and their carers in health and social care. NICE clinical guidelines, 2006 (amended 2011):35-7.

3. American Psychiatric Association. Practice guideline for the treatment of patients with Alzheimer's disease and other dementias. 2nd edn. 2007:31.

4. Gentile S. Second-generation antipsychotics in dementia: beyond safety concerns. A clinical, systematic review of efficacy data from randomised controlled trials. Psychopharmacology (Berl) 2010;212:119-29.

5. Ballard C, Waite J. The effectiveness of atypical antipsychotics for the treatment of aggression and psychosis in Alzheimer's disease. Cochrane Database Syst Rev 2006;(1):CD003476.

6. Schneider LS, Dagerman KS, Insel P. Risk of death with atypical antipsychotic drug treatment for dementia: meta-analysis of randomized placebo-controlled trials. JAMA 2005;294:1934-43.

7. Schneider LS, Dagerman K, Insel PS. Efficacy and adverse effects of atypical antipsychotics for dementia: meta-analysis of randomized, placebo-controlled trials. Am J Geriatr Psychiatry 2006;14:191-210.

8. Ballard C, Lana MM, Theodoulou M, et al. A randomised, blinded, placebo-controlled trial in dementia patients continuing or stopping neuroleptics (the DART-AD trial). PLoS Med 2008;5:e76.

9. US Food and Drug Administration. FDA Requests Boxed Warnings on Older Class of Antipsychotic Drugs. Secondary FDA Requests Boxed Warnings on Older Class of Antipsychotic Drugs. 2008. http:// www.fda.gov/NewsEvents/Newsroom/PressAnnouncements/2008/ ucm116912.htm

10. Kales HC, Zivin K, Kim HM, et al. Trends in antipsychotic use in dementia 1999-2007. Arch Gen Psychiatry 2011;68:190-7.

11. Snowdon J, Galanos D, Vaswani D. A 2009 survey of psychotropic medication use in Sydney nursing homes. Med $J$ Aust 2011;194: 270-1.

12. Banergee S. The use of antipsychotic medication for people with dementia: time for action. A report for the Minister of State for Care Services, 2009.

13. Walker FO. Huntington's disease. Lancet 2007;369:218-28.

14. MacMillan JC, Harper PS. Single-gene neurological disorders in South Wales: an epidemiological study. Ann Neurol 1991;30:411-14.

15. Vamos M, Hambridge J, Edwards M, et al. The impact of Huntington's disease on family life. Psychosomatics 2007;48:400-4.

16. Craufurd G, Snowden J. Neuropsychological and neuropsychiatric aspects of Huntington disease. In: Bates G, Harper PS, Jones L, eds. Huntington's disease. 3rd edn. Oxford: Oxford University Press, 2002:62-94.

17. YPINH National Alliance. 2011. http://www.ypinh.org.au/index.php/ Statistics.htm; Secondary http://www.ypinh.org.au/index.php/ Statistics.htm. http://www.ypinh.org.au/index.php/Statistics.htm

18. Kaufer DI, Cummings JL, Ketchel P, et al. Validation of the NPI-Q, a brief clinical form of the Neuropsychiatric Inventory. J Neuropsychiatry Clin Neurosci 2000;12:233-9.

19. Cane J, O'Connor D, Michie S. Validation of the theoretical domains framework for use in behaviour change and implementation research. Implement Sci 2012;7:37.

20. Woods SW. Chlorpromazine equivalent doses for the newer atypical antipsychotics. J Clin Psychiatry 2003;64:663-7. 